\title{
Global Sensitivity Analysis for Uncertain Parameters Applied to a Cardiac Mitochondrial Model
}

\author{
Bachar Tarraf ${ }^{1,2,3}$, Michael Leguèbe ${ }^{1,2,3}$, Yves Coudière ${ }^{4,3,1,2}$, Emmanuel Suraniti $^{5}$, Camille Colin $^{5,2}$, \\ Stephane Arbault ${ }^{5}$, Philippe Diolez ${ }^{2,4,6}$ \\ ${ }^{1}$ Inria Bordeaux-Sud-Ouest team CARMEN, Talence, France \\ ${ }^{2}$ IHU Liryc, Fondation Bordeaux Université, Pessac, France \\ ${ }^{3}$ Institut de Mathématiques de Bordeaux UMR CNRS 5251, Talence, France \\ ${ }^{4}$ Université de Bordeaux, Bordeaux, France \\ ${ }^{5}$ Univ. Bordeaux, CNRS, Bordeaux INP, ISM, UMR 5255, F-33400 Talence, France \\ ${ }^{6}$ INSERM, Centre de recherche Cardio-Thoracique de Bordeaux, U1045, Bordeaux, France
}

\begin{abstract}
Cardiac mitochondria are intracellular organelles that have several important roles. For instance, they ensure energy metabolism and calcium regulation, thus are linked to the excitation-contraction cycle of the heart cell. Mathematical models are useful to better understand the complexity of mitochondrial dynamics within a cardiac cell, and we are specifically interested in the dynamics of calcium. Litterature models reflect this complexity, especially in terms of number of equations and parameters, which makes them impossible to calibrate to experimental data.

In this paper, we apply a global sensitivity analysis on our previously discussed simple mitochondria model [1], in order to quantify the uncertainty of the parameters. This analysis is done in two steps. First we eliminate noninfluential parameters of the internal fluxes governing the activity of the mitochondria. Then we perform this analysis on the outputs of our ODE (ordinary differential equation) model, which are the respiratory rates.

Finally, we calibrate the remaining influential parameters using a genetic algorithm with respect to experimental respiratory data.
\end{abstract}

\section{Introduction}

It is widely known that cardiac contraction is triggered by calcium $\left(\mathrm{Ca}^{2+}\right)$, hence a perturbation in the intracellular $\mathrm{Ca}^{2+}$ homeostasis could lead to heart failure [2]. Cardiac mitochondria are intracellular organelles that participate in the $\mathrm{Ca}^{2+}$ regulation through their channels or pores: $\mathrm{Ca}^{2+}$ uniporter, $\mathrm{Na}^{+} / \mathrm{Ca}^{2+}$ exchanger and mitochondria permeability transition pore (mPTP). For this reason, developping cardiac models that take into account the activ- ity of the mitochondria is important. In our previous paper [1], we introduced a mathematical model that describes key mechanisms of the cardiac mitochondria, specifically dioxygen consumption, calcium $\left(\mathrm{Ca}^{2+}\right)$ regulation through the uniporter and the $\mathrm{Na}^{+} / \mathrm{Ca}^{2+}$ exchanger, and the ATP synthesis. The model consists of six state variables that describe the activity of the mitochondria through ion fluxes (Fig,1). These fluxes model the variation of the state variables in time. We recall the model's differential equations

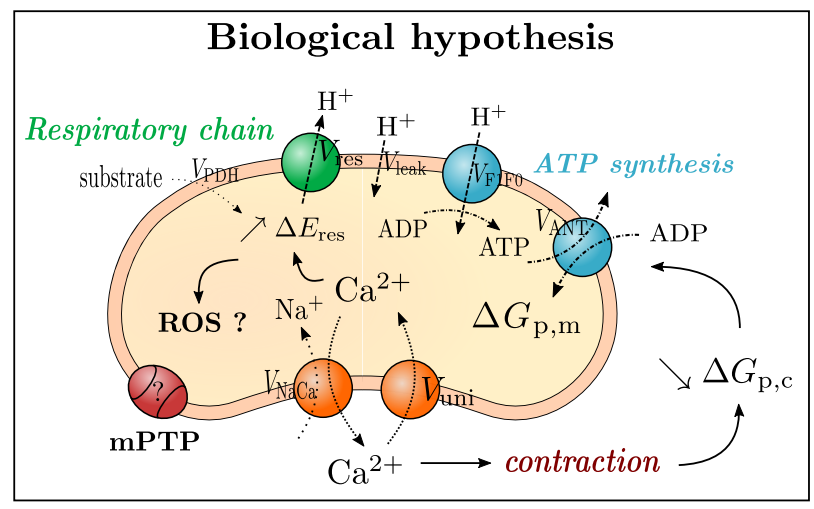

Figure 1. Reactions and fluxes described by the model. State variables are indicated by bold font.

system $(\mathrm{S})$ :

$$
\begin{aligned}
& \partial_{t} \Delta E_{\mathrm{resp}}=\varphi_{1}\left(\Delta E_{\mathrm{resp}}\right)\left(V_{\mathrm{PDH}}-V_{\text {resp }}\right), \\
& \partial_{t} \Delta G_{\mathrm{p}, \mathrm{m}}=\varphi_{2}\left(\Delta G_{\mathrm{p}, \mathrm{m}}\right)\left(V_{\mathrm{F} 1 \mathrm{~F} 0}-V_{\mathrm{ANT}}\right), \\
& \partial_{t} \Delta G_{\mathrm{p}, \mathrm{c}}=\varphi_{3}\left(\Delta G_{\mathrm{p}, \mathrm{c}}\right)\left(V_{\mathrm{ANT}}+\dot{g}_{\text {ext }}(t)\right), \\
& \partial_{t} \Delta p=\left(12 V_{\mathrm{resp}}-3 V_{\mathrm{F} 1 \mathrm{~F} 0}-V_{\mathrm{ANT}}-V_{\text {leak }}-2 V_{\mathrm{uni}}\right) / C_{\mathrm{m}}, \\
& \partial_{t}\left[\mathrm{Ca}^{2+}\right]_{\mathrm{m}}=f\left(V_{\mathrm{uni}}-V_{\mathrm{NaCa}}\right), \\
& \partial_{t}\left[\mathrm{Ca}^{2+}\right]_{\mathrm{c}}=-\gamma \partial_{t}\left[\mathrm{Ca}^{2+}\right]_{\mathrm{m}}+\dot{c}_{\mathrm{ext}}(t),
\end{aligned}
$$


where the functions $\varphi_{i}$ are known and the parameters $f$ and $\gamma$ account for the fraction of free $\mathrm{Ca}^{2+}$ in the matrix and in the cytosol, respectively. The functions $\dot{g}_{\text {ext }}(t)$ and $\dot{c}_{\text {ext }}(t)$ are source terms for cytoplasmic ADP and $\mathrm{Ca}^{2+}$, respectively. These source terms allow us to mimic an experimental condition, for instance an addition of cytoplasmic ADP. The biological meaning of the fluxes $V_{j}$ and the derivation of their expressions can be found in [1].

Even though our model is considered simple (34 parameters overall) in comparison with models in the literature, it is still impossible to calibrate it to fit experimental data. For this reason, we first perform a global sensitivity analysis to eliminate parameters wich have small influence on these fluxes (sec. 2.1. Then, using the results from this first step, we perform again the analysis, but this time on the observables of our model as output (sec. 2.2). Finally (sec. 3), we use the results of the sensitivity analysis to calibrate the few remaining parameters to experimental respiratory rates.

\section{Global sensitivity analysis}

The concept of global sensitivity analysis is to quantify the influence of a certain parameter on the output of a model, while spanning the whole parameter space, that can be of very high-dimension. In practice, we used the Sobol analysis method which consists in decomposing the variance of the output of a model with respect to its parameters [3]. Beside being able to explore globally the parameter space, this method can also take into account the statistical distribution of the parameters.

Typically, a model is written as $Y=f\left(p_{1}, p_{2}, \ldots, p_{k}\right)$ where $\mathrm{Y}$ is the output and $p_{i}, i \in\{1, \ldots, k\}$ are the parameters. For each parameter $p_{i}$, the total Sobol index associated to $p_{i}$ is $S_{T_{i}}=\frac{E\left[V\left(Y / p_{\sim i}\right)\right]}{V(Y)}=1-\frac{V\left(E\left[Y / p_{\sim i}\right]\right)}{V(Y)}$, where $p_{\sim i}$ denotes all the parameters except $p_{i}$. The less influential the parameter $p_{i}$ is on the output $Y$, the closer to zero the index $S_{T_{i}}$ is, and vice versa. If $S_{T_{i}} \simeq 0$, then $p_{i}$ can be fixed in its range of variability without significantly affecting $V(Y)$.

The straightforward approach for calculating the Sobol index associated to a parameter $p_{i}$ is computationally expensive. To overcome this, we used the Saltelli sampling method [3] which reduces the total number of evaluations of $f$ to $N \times(k+2)$, where $N$ is the size of the parameters sample. All the Sobol indices were computed using the SALib Python library [4].

\subsection{Sobol's analysis on fluxes}

As a first step of our sensitivity analysis, each flux $V_{j}$ is considered as a seperate model that depends on some parameters. Computing the Sobol indices of a parameter gives an idea of its influence on the associated flux. The

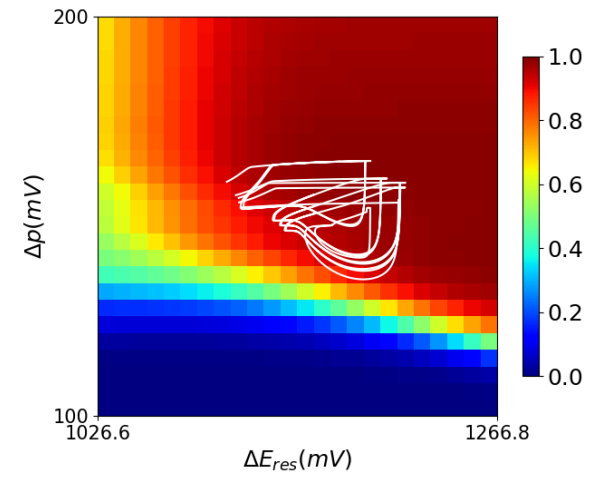

Figure 2. Total Sobol indices of parameter $p_{2}$ for the flux $V_{\text {resp. }}$. White lines locate several trajectories in the phase space $\left(\Delta E_{\text {resp }}, \Delta p\right)$. Color depicts the magnitude of the Sobol index. Trajectories were generated using random samples of the whole set of parameters.

fluxes are not only functions of their associated parameters, but also of the state variables, and hence computing the Sobol indices for a large range of state space points is necessary.

For each flux, to be able to eliminate uninfluential parameters, we checked that the trajectories of the state variables along typical mitochondrial respiratory cycles did not cross areas with hight Sobol indices (Fig. 2 for an example of an influential parameter). Using this method, we were able to eliminate 7 parameters out of 27 involved in the expression of the fluxes, among the overall 34 parameters.

\subsection{Sobol's analysis on the output of (S)}

The ODE system (S) can be seen as an equation $y=$ $g(p)$, where $p$ is the set of parameters, and $y$ is the solution over the integration time interval. To have a global idea on the influence of the remaining set of parameters $\tilde{p}$ on the solution $y$, we perform the Sobol analysis on the model $y=\tilde{g}(\tilde{p})$, where $\tilde{g}$ is the model function $g$ with the 7 previously identified parameters fixed in their range of uncertainty.

Each evaluation of $\tilde{g}$ requires an ODE system to be solved accurately, which can be problematic as the system includes very fast dynamics and the source term $\dot{g}_{\text {ext }}$ has a stiff profile. A predictor-corrector numerical scheme allowed for integration of the equations in $\sim 100$ seconds. With $k=\operatorname{Card}(\tilde{p})=34-7=27$ parameters, and using a sampling number $n=2000$, then 58000 model evaluations were required. Computations were performed in parallel using the PlaFRIM experimental testbed. The results are presented on figure 3 


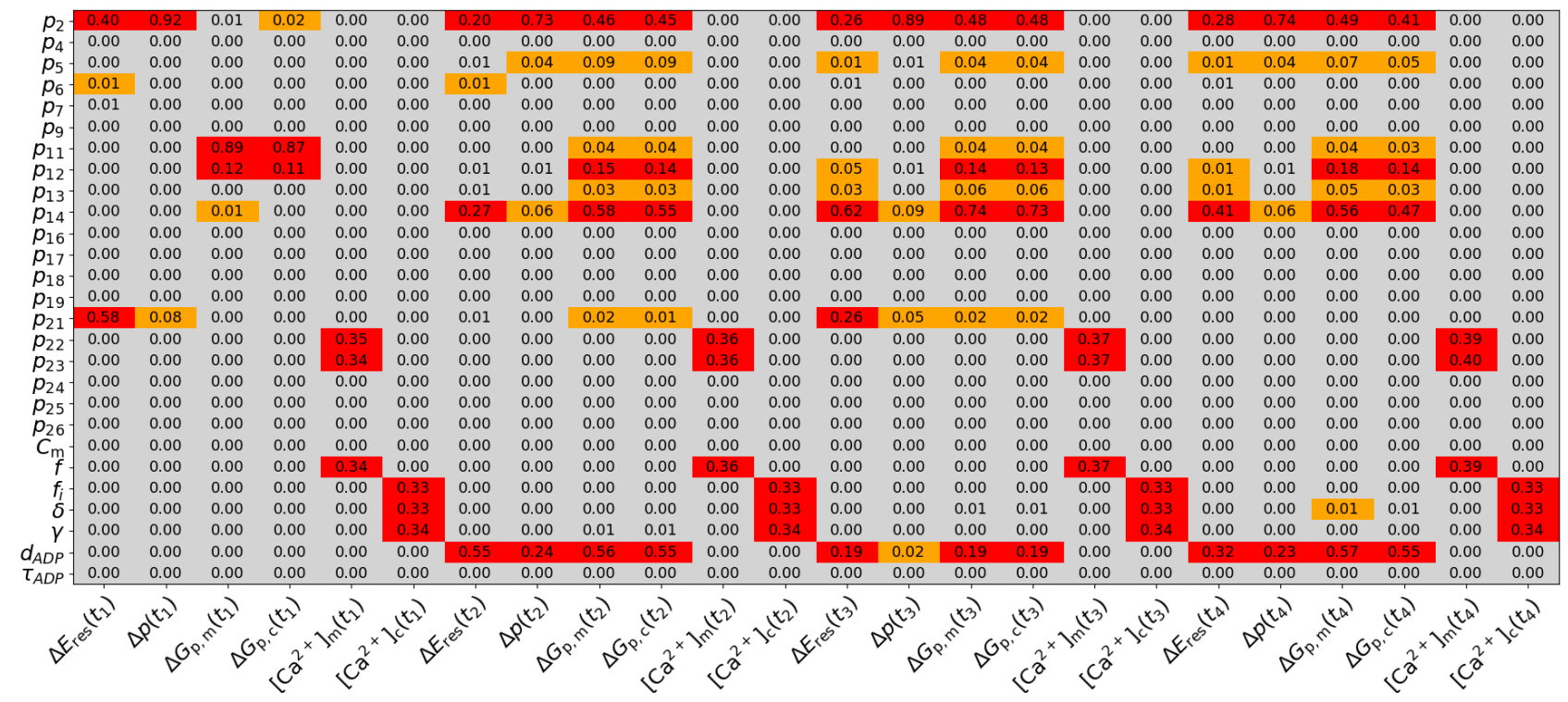

Figure 3. Total Sobol index for the set of parameters $q$ associated to the system (S). The output of our model is considered at 4 different time points, $t_{1}$ (before first addition), $t_{2}$ (after first addition), $t_{3}$ (before second additon), $t_{4}$ (after second addition). First addition is $0.66 \mathrm{mM}$ cytoplasmic ADP, second addition is $1 \mathrm{mM}$ cytoplasmic ADP. The 3 colors level represent the magnitude of the total Sobol index, grey being the lowest $\left(S_{T}<10^{-2}\right)$, then yellow $\left(10^{-2} \leq S_{T}<10^{-1}\right)$, and orange being the highest $\left(S_{T} \geq 10^{-1}\right)$.

\section{Parameters calibration}

\subsection{Experimental data}

The experimental data were obtained from heart mitochondria extracted from Wistar Male rats (from Janviers Lab, France). Isolated mitochondria were analyzed within the day following their preparation and subsequent determination of proteins concentration by the Bratford method. All effectors of the respiratory chain used herein were first prepared as concentrated mother solutions $(500 \mathrm{mM}$ for the substrates of the respiratory chain, glutamate and malate, and $100 \mathrm{mM}$ for ADP; all compounds were purchased from Sigma-France) in the specific respiration buffer for mammal heart mitochondria. All solutions were kept on ice during the timescales of experiments.

The mitochondrial oxygen consumption rates were collected by the gold standard method in bioenergetics, that is chronoamperometry with a Clark electrode [5]. This electrochemical system determines the dissolved oxygen concentration of a liquid medium by measuring the current resulting from the 4-electron reduction of dioxygen $\mathrm{O}_{2}$ to water $\mathrm{H}_{2} \mathrm{O}$ on a platinum disk (at a potential of $-0.8 \mathrm{~V}$ vs the internal $\mathrm{Ag} / \mathrm{AgCl}$ reference electrode). $\mathrm{O}_{2}$ diffuses towards the platinum through a Teflon membrane protecting it from the solution. The Clark electrode was inserted in an oxygraphy chamber filled with $6 \mathrm{~mL}$ of respiration buffer under constant stirring, and thermostated at $28{ }^{\circ} \mathrm{C}$ with a water gasket. Substrates of the respiratory chain were injected at $5 \mathrm{mM}$ final concentration, then mitochondria were injected to reach a concentration of 0.4 mgprot. $\mathrm{mL}^{-1}$ in the respiration buffer. ADP was further introduced at various concentrations ( 0.166 to $1 \mathrm{mM})$. The current at the Clark electrode was continuously measured, and the oxygen concentration determined by a simple proportionality factor $\left(210 \mu \mathrm{M}\right.$ of $\mathrm{O}_{2}$ maximum concentration).

\subsection{Optimization algorithm}

As observed on Fig. 3, we found that 6 parameters $\left\{p_{2}, p_{11}, p_{12}, p_{14}, p_{21}, d_{A D P}\right\}$ are influential on the respiratory rates. The remaining parameters with a high Sobol index are not considered, since they would be rather related to Calcium fluxes. We choose to apply a genetic optimization algorithm [6] to fit these parameters to the data, because of the high dimensionality of the problem. The selection process at each iteration is based on minimizing $\left\|y_{\text {sim }}-y_{\text {obs }}\right\|_{l^{2}}$, where $y_{\text {obs }}$ is the experimental normalized oxygen concentration, and $y_{\text {sim }}$ is the simulated one. From the selected set of parameters, we apply the mutation and crossover operators to eventually obtain the next generation of parameters that is trivially better adapted to our problem. The algorithm allowed convergence to an optimum set of parameters after 25 iterations. Figure 4 shows the resulting oxygen concentration over the time compared to the experimental data. 


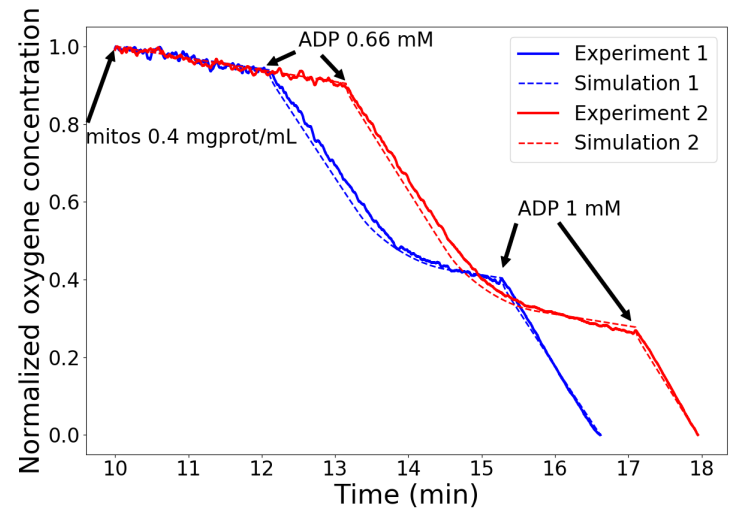

Figure 4. Comparison of the oxygen consumption between two sets of experimental data, in the presence of $5 \mathrm{mM}$ of glutamate and malate, and simulation, for the substrate-state (before ADP addition) and phosphorylating state 3 (after ADP addition) of the mitochondria.

\section{Discussion}

The Sobol sensitivity analysis on fluxes revealed only 7 uninfluential parameters from the starting set of 27 flux parameters. This indicate that our choices of expressions for the fluxes is nearly optimal (ie minimizes the number of parameters).

However the Sobol analysis on the whole model highlighted only very few parameters as influencing the respiratory rates. We also noticed in figure 3 a block structure that discriminates parameters which influence calcium concentrations, from the ones which influence the respiratory rates. It suggests that further calibration of these subsets can be performed separately.

All Sobol indices were less than $10^{-1}$ except for the parameters $p_{2}, p_{11}, p_{12}, p_{14}, p_{21}$, and $d_{A D P}$, for which total Sobol indices reach $0.92,0.89,0.18,0.74,0.58$, and 0.57 , respectively. The first parameter controls the maximum amplitude of the respiratory rate $V_{\text {resp }}$. The parameters $p_{11}, p_{12}$, and $p_{14}$ control the ATP/ADP and $\Delta p$ dependent activation threshold of the ATP-synthase, respectively. The parameter $p_{21}$ tunes the amplitude of the proton leak across the mitochondrial membrane.The corresponding high Sobol index before any ADP addition $\left(S_{T}=\right.$ 0.58 ) is thus expected, since at this stage mitochondria are only respiring to compensate the proton leakage. Finally, the parameter $d_{A D P}$ which controls the duration of the addition of ADP has a high Sobol index which can also be expected.

The optimization algorithm converges towards an optimal set for these parameters. The relative $l_{2}$ error with respect to the data is less than 0.032 (Fig. 4). It shows the ability of our model to reproduce experimental respiratory rates in both phosphorylating state 3 and substrate-state of the mitochondria.

\section{Conclusion}

In this work, we firstly carried out a global sensitivity analysis on the model proposed in [1]. It was completed in two steps, the analysis on the internal mitochondrial flux functions outputs, and the analysis on the state variables of the model along a typical trajectory. It allowed to determine the main relevant parameters. Secondly, we applied an iterative optimization algorithm to succesfully calibrate these six parameters to a given experimental situation. We expect repeated sentivity analysis with diverse experimental data (e.g. calcium, reactive oxygen species) to allow for more parameters to be calibrated.

\section{Acknowledgments}

This study received financial support from the French Government as part of the "Investments of the Future" program managed by the Agence Nationale de la Recherche grant ANR-10-IAHU-04 and also from the project MITOCARD (ANR-17-CE11-0041). Some of the experiments presented in this paper were carried out using the PlaFRIM experimental testbed, supported by Inria, CNRS (LaBRI and IMB), Université de Bordeaux, Bordeaux INP and Conseil Régional d'Aquitaine (see https://www.plafrim.fr/).

\section{References}

[1] Tarraf B, Leguèbe M, Coudière Y, Diolez P. Thermodynamical fluxes for the modeling of cardiac mitochondrial calcium handling. In Computing In Cardiology. Singapore, 2019;

[2] Santulli G, Xie W, Reiken SR, Marks AR. Mitochondrial calcium overload is a key determinant in heart failure. Proceedings of the National Academy of Sciences 2015; 112(36):11389-11394.

[3] Saltelli A, Ratto M, Andres T, Campolongo F, Cariboni J, Gatelli D, Saisana M, Tarantola S. Global sensitivity analysis: the primer. John Wiley \& Sons, 2008.

[4] Jon H, Will U. SALib: An open-source python library for sensitivity analysis. J Open Source Softw jan 2017;2(9). URL https://doi.org/10.21105/joss.00097

[5] Palmeira CM, Moreno AJ (eds.). Mitochondrial Bioenergetics, volume 1782 of Methods in Molecular Biology. Humana Press, 2008.

[6] Durand N. Algorithmes Génétiques et autres méthodes d'optimisation appliqués à la gestion de trafic aérien. Ph.D. thesis, 2004.

Address for correspondence:

Bachar Tarraf

Inria Bordeaux-Sud-Ouest

200 avenue de la Vieille Tour

bachar.tarraf@inria.fr 